\title{
A national alcohol strategy for Chile: Rationale, development, content and status of implementation
}

\author{
Norman Giesbrecht ${ }^{1,2}$, Jaime C. Sapag ${ }^{1,3}$, Alfredo Pemjean ${ }^{4,5}$, Judith Marquez ${ }^{1}$ Akwatu Khenti ${ }^{1}$, \\ Jürgen Rehm ${ }^{1-3,6,7}$, and Alberto Minoletti ${ }^{8}$ \\ ${ }^{1}$ Centre for Addiction and Mental Health \\ ${ }^{2}$ Dalla Lana School of Public Health, University of Toronto, Toronto, Canada \\ ${ }^{3}$ Department of Psychiatry, University of Toronto, Toronto, Canada \\ ${ }^{4}$ Department of Psychiatry and Mental Health, Faculty of Medicine, Universidad Diego Portales, Chile \\ ${ }^{5}$ Department of Mental Health, Ministry of Health, Santiago, Chile \\ ${ }^{6}$ Institute of Medical Science, University of Toronto, Toronto, Canada \\ ${ }^{7}$ Technische Universitaet Dresden, Dresden, Germany \\ ${ }^{8}$ School of Public Health, University of Chile, Santiago, Chile
}

\section{Abstract}

Aim: This paper describes the rationale for the Chilean strategy on alcohol, how it was developed, its key recommendations, which of its dimensions have been implemented, and remaining challenges.

Design: The paper is based on archival data, a literature review, and survey data from Chilean sources. It draws on presentations at two seminars in Santiago, and a background document commissioned by the Chilean Ministry of Health. Building on ongoing initiatives in Chile, it was informed by international research on the global and regional burden of disease from alcohol.

Setting and Context: In 2008 the Ministry of Health, Government of Chile, embarked on developing a national alcohol strategy. The strategy's rationale was informed by the high rate of alcohol-related trauma, including drinking and driving; the high rate of liver cirrhosis mortality and morbidity; the high rate of heavy drinking, including among youth and young adults; and gender differences.

Measures: The main recommendations focused on several themes: pricing and taxation interventions, controlling physical availability, curtailing alcohol marketing, promoting server intervention, controlling drinking and driving, promoting communitybased interventions, facilitating screening and brief interventions, and monitoring and tracking local and national developments on alcohol issues.

Findings \& Conclusions: Since 2008, there has been progress in several areas, including a National Strategy for brief interventions in primary care; a new law on legal blood alcohol content; proposals to increase taxes on spirits, introduce warning labels on beverage containers, and limit promotion of alcoholic beverages; and the integration of alcohol-related goals within the National Health Strategy 2011-2020. Nevertheless, challenges remain: the broad acceptance of drinking, including high-risk drinking; the importance and influence of the alcohol industry; and the need for an evidence-based inter-sector response.

The harmful use of alcohol has a detrimental effect on public health and safety, and it also represents a social and economic development issue worldwide. Alcohol-related challenges are particularly important in the Americas (Rehm \& Monteiro, 2005), where the problem surpasses average global statistics (Monteiro, 2007)'in terms of consumption, risky drinking patterns, and disorders, and as a risk factor for disease and mortality.

The harm associated with alcohol can be ameliorated if effective actions are taken by countries to protect their populations. Comprehensive and effective alcohol policies and strategies are needed to address the alcohol problem (Anderson, Chisholm, \& Fuhr, 2009; Babor et al., 2010; Brand, Saisana, Ryan, Pennoni, \& Lowenfels, 2007). A wide range of public health-oriented strategies are required for health promotion, prevention and treatment (Babor et al., 2010; Giesbrecht, Stockwell, Kendall, Strang, \& Thomas, 2011); however, interventions and policies typically are not keeping pace with the burden of financial and social costs.

In 2010, the World Health Organization launched the Global Strategy on Alcohol (World Health Organization

Correspondence: Norman Giesbrecht, Ph.D., Social \& Epidemiological Research Dept., Centre for Addiction \& Mental Health, CAMH 33 Russell St. Toronto, Ontario, Canada M5S 2S1

Telephone 416-535-8501 ext 6895. Fax 416 595-6899. email: norman_giesbrecht@camh.ca

Keywords: alcohol policy, Chile, regulation, public health approach 
[WHO], 2010) to reduce the harmful use of alcohol, calling for the development of adequately funded, evidence-based, comprehensive and inter-sector national policies. That strategy identifies several areas where interventions are needed, highlighting the importance of tailoring the process to local circumstances and defining clear objectives, strategies and targets, involving key stakeholders. Countries in Latin America have taken initiatives that complement this 2010 WHO strategy (Babor \& Caetano, 2005; Hospedales et al., 2012).

Chile has been one of the leading countries in that regard. A process to develop a National Alcohol Strategy was implemented between 2008 and 2010. The national strategy built on ongoing initiatives in Chile and was informed by international research, including research on the contribution of alcohol to the global and regional burden of disease (Babor et al., 2003), as well as publications and recommendations from $\mathrm{PAHO} / \mathrm{WHO}$ (Monteiro, 2007; WHO, 2010).

\section{Purpose and Methods}

The purpose of this article is to describe and analyze the rationale for the national alcohol strategy in Chile, including how it was developed and the key recommendations. The article also outlines which dimensions have been implemented since the strategy was introduced, and discusses key challenges related to implementing and sustaining effective alcohol policies in Chile.

The paper is based on several sources, including archival data, a literature review, and survey data from Chilean documents. It also draws on presentations at a June 2009 seminar in Santiago and a subsequent seminar in December 2010. A seminal resource is the background document commissioned by the Chilean Ministry of Health to the Centre for Addiction and Mental Health (CAMH), and coauthored by Giesbrecht, Marquez, and Sapag (2009).

\section{Results}

\section{The Chilean Context and the Rational for an Alcohol Strategy}

The patterns of alcohol consumption and alcohol-related public health problems by 2008-2009 were part of the context for this initiative. During this time, several persons in the government became aware of the magnitude and range of these problems, which include traffic accidents associated with alcohol use and disability-adjusted life years (DALYs) related to alcohol consumption. In planning a response for Chile, they drew on reliable international information on the impact of public policies on alcohol consumption and alcohol-related harm.

The recorded 2007 per-adult (aged 15 or over) sale of beer was 33.4 liters (beverage volume) or about 1.50 liters in absolute alcohol (4.5\% strength). For 2006, the beverage volume of wine was 18.84 per capita, or about 2.26 liters in absolute alcohol (12\% strength). In Table 1 we provide trends in estimated per-adult consumption for 1998 to 2012, based on official sales and estimates of unrecorded consumption. Recorded per-adult consumption increased gradually between 2003 and 2012. If both registered alcoholic beverages and estimated non-registered alcohol are considered, the consumption for 2012 was 9.8 liters pure alcohol per person aged 15 or over (Table 1 ).

Table 1

Estimated alcohol consumption in liters of pure alcohol, per capita ages 15 and older, Chile. World Survey Report, 2012

\begin{tabular}{|c|c|c|c|c|c|c|c|c|}
\hline \multirow[b]{2}{*}{ Year } & \multicolumn{2}{|c|}{ Population } & \multicolumn{3}{|c|}{$\begin{array}{c}\text { Consumption per capita, by } \\
\text { beverage type }\end{array}$} & \multirow[b]{2}{*}{$\begin{array}{c}\text { Consumption } \\
\text { per capita (all } \\
\text { beverage types) }\end{array}$} & \multirow[b]{2}{*}{$\begin{array}{c}\text { Unrecorded } \\
\text { per capita } \\
\text { consumption }\end{array}$} & \multirow[b]{2}{*}{$\begin{array}{c}\text { Total per } \\
\text { capita } \\
\text { consumption }\end{array}$} \\
\hline & $\begin{array}{c}\text { Total } \\
\text { Population }\end{array}$ & $\begin{array}{c}\text { Population } 15 \\
\text { years and } \\
\text { older }\end{array}$ & Wine & Beer & Spirits & & & \\
\hline 1998 & $14,996,647$ & $10,720,577$ & 3.03727 & 2.10183 & 2.33801 & 7.47711 & 2.0 & 9.5 \\
\hline 1999 & $15,197,213$ & $10,919,190$ & 3.13537 & 1.89237 & 2.01602 & 7.04376 & 2.0 & 9.0 \\
\hline 2000 & $15,397,784$ & $11,117,809$ & 2.45141 & 1.75053 & 2.22145 & 6.42338 & 2.0 & 8.4 \\
\hline 2001 & $15,571,679$ & $11,336,882$ & 2.38218 & 1.91538 & 2.43325 & 6.73081 & 2.0 & 8.7 \\
\hline 2002 & $15,745,583$ & $11,555,963$ & 2.38584 & 1.88472 & 1.62488 & 5.89545 & 2.0 & 7.9 \\
\hline 2003 & $15,919,479$ & $11,775,038$ & 2.60043 & 2.28073 & 2.46117 & 7.34232 & 2.0 & 9.3 \\
\hline 2004 & $16,093,378$ & $11,994,115$ & 2.54864 & 1.80563 & 2.82031 & 7.17457 & 2.0 & 9.2 \\
\hline 2005 & $16,267,278$ & $12,213,191$ & 2.59375 & 2.01004 & 2.97134 & 7.57513 & 2.0 & 9.6 \\
\hline 2006 & $16,432,674$ & $12,426,455$ & 2.21666 & 2.26456 & 2.85163 & 7.33285 & 2.0 & 9.3 \\
\hline 2007 & $16,598,074$ & $12,639,720$ & 2.83471 & 2.33716 & 2.21740 & 7.38928 & 2.0 & 9.4 \\
\hline 2008 & $16,763,470$ & $12,852,982$ & 2.17282 & 2.43433 & 2.70966 & 7.31682 & 2.0 & 9.3 \\
\hline 2009 & $16,928,873$ & $13,066,251$ & 2.84519 & 2.32226 & 2.50162 & 7.66907 & 2.0 & 9.7 \\
\hline 2010 & $17,094,275$ & $13,279,518$ & 3.23092 & 2.36512 & 2.32742 & 7.92346 & 2.0 & 9.9 \\
\hline 2011 & $17,248,450$ & $13,447,604$ & 2.69965 & 2.41026 & 2.36389 & 7.47380 & 2.0 & 9.5 \\
\hline 2012 & $17,402,630$ & $13,615,694$ & 2.77621 & 2.45429 & 2.61074 & 7.84124 & 2.0 & 9.8 \\
\hline
\end{tabular}


Table 2

Percent of respondents who reported consuming alcohol in the past month, Chile, 1994-2010

\begin{tabular}{|c|c|c|c|c|c|c|c|c|c|c|c|}
\hline \multirow[b]{2}{*}{ Year } & \multirow[b]{2}{*}{ Total } & \multicolumn{2}{|c|}{ Gender } & \multicolumn{5}{|c|}{ Age Group } & \multicolumn{3}{|c|}{ Socio-economic status } \\
\hline & & Male & Female & $12-18$ & $19-25$ & $26-34$ & $35-44$ & $45-64$ & Low & Medium & High \\
\hline 1994 & 40.4 & 50.6 & 31.0 & 24.4 & 49.4 & 46.7 & 43.7 & 36.5 & 38.1 & 38.1 & 47.0 \\
\hline 1996 & 48.1 & 56.9 & 39.8 & 31.4 & 56.7 & 52.3 & 51.5 & 46.6 & $\mathrm{sd}^{* *}$ & $\mathrm{sd}^{* *}$ & $\mathrm{sd}^{* *}$ \\
\hline 1998 & 53.0 & 64.0 & 42.5 & 30.9 & 60.3 & 58.3 & 57.9 & 54.4 & 49.1 & 52.8 & 55.3 \\
\hline 2000 & 54.4 & 63.4 & 45.8 & 30.6 & 61.1 & 60.5 & 59.8 & 57.2 & 51.6 & 53.2 & 60.2 \\
\hline 2002 & 59.6 & 66.8 & 52.8 & 35.5 & 65.8 & 66.6 & 65.9 & 61.7 & 58.2 & 59.3 & 62.5 \\
\hline 2004 & 57.9 & 66.6 & 49.4 & 31.5 & 65.4 & 65.3 & 63.0 & 61.2 & 55.3 & 57.1 & 63.2 \\
\hline 2006 & 58.1 & 65.9 & 50.5 & 32.3 & 64.0 & 65.9 & 63.9 & 61.0 & 53.9 & 58.1 & 64.2 \\
\hline 2008 & 49.8 & 60.0 & 39.9 & 27.2 & 60.8 & 60.8 & 53.7 & 47.1 & 46.2 & 49.3 & 55.9 \\
\hline 2010 & $40.5^{*}$ & $48.9 *$ & $39.2^{*}$ & $18.4^{*}$ & 55.4 & $47.7^{*}$ & $44.4^{*}$ & $39.0 *$ & $35.2 *$ & $37.3^{*}$ & $47.1^{*}$ \\
\hline
\end{tabular}

*Statistically significant variation in respect to the previous study.

**The question about "quality of household" was not included to build the socio-economic status variable.

Source: CONACE (2010).

Table 3

Percent of respondents who reported consuming alcohol in the past year, Chile, $1994-2010$

\begin{tabular}{|c|c|c|c|c|c|c|c|c|c|c|c|}
\hline \multirow[b]{2}{*}{ Year } & \multirow[b]{2}{*}{ Total } & \multicolumn{2}{|c|}{ Gender } & \multicolumn{5}{|c|}{ Age Group } & \multicolumn{3}{|c|}{ Socio-economic status } \\
\hline & & Male & Female & $12-18$ & $19-25$ & $26-34$ & $35-44$ & $45-64$ & Low & Medium & High \\
\hline 1994 & 60.9 & 69.0 & 53.5 & 43.3 & 69.7 & 69.1 & 63.2 & 57.7 & 57.0 & 61.7 & 67.9 \\
\hline 1996 & 71.5 & 77.9 & 65.4 & 56.0 & 78.0 & 78.1 & 76.0 & 67.5 & $\mathrm{sd}^{* *}$ & $\mathrm{sd}^{* *}$ & $\mathrm{sd}^{* *}$ \\
\hline 1998 & 71.6 & 79.9 & 63.7 & 52.4 & 79.1 & 77.2 & 76.5 & 70.7 & 68.1 & 70.7 & 74.0 \\
\hline 2000 & 73.9 & 79.4 & 68.7 & 53.8 & 81.7 & 80.2 & 79.0 & 73.4 & 71.7 & 73.0 & 78.4 \\
\hline 2002 & 75.3 & 79.6 & 71.2 & 55.8 & 83.1 & 81.8 & 79.8 & 75.0 & 73.5 & 75.1 & 78.4 \\
\hline 2004 & 76.0 & 80.9 & 71.2 & 55.5 & 83.7 & 82.2 & 80.1 & 77.0 & 73.8 & 75.4 & 80.3 \\
\hline 2006 & 73.6 & 78.4 & 68.8 & 48.0 & 80.0 & 82.4 & 79.3 & 75.3 & 70.2 & 74.3 & 77.5 \\
\hline 2008 & 68.5 & 75.6 & 61.6 & 46.2 & 77.9 & 78.1 & 71.5 & 68.0 & 65.7 & 69.7 & 70.8 \\
\hline 2010 & $57.5^{*}$ & $63.7^{*}$ & $51.4^{*}$ & $31.1^{*}$ & $71.5^{*}$ & $65.6^{*}$ & $62.0 *$ & $58.0^{*}$ & $58.8^{*}$ & 54.3 & $60.5^{*}$ \\
\hline
\end{tabular}

*Statistically significant variation in respect to the previous study.

**The question about "quality of household" was not included to build the socio-economic status variable.

Source: CONACE (2010).

It should be noted that the WHO-projected estimates for 2008 indicate 8.8 for recorded and unrecorded consumption combined-somewhat lower than the 9.3 shown in Table 1 -and the Global Status Report on Alcohol and Health indicates 8.55 for Chile (unrecorded and recorded) (WHO, 2011).

Survey data show that the prevalence of alcohol consumers among Chileans grew continuously from 1994 to 2002, and then declined thereafter (Tables 2 and 3). Males showed a higher prevalence for all periods between 1994 and 2010 for percent drinking in the past month, and also past year (Table 2 and Table 3). In 2006, 66\% of men and 51\% of women reported drinking in the past month (Table 2).
Between 1994 and 2006 the prevalence of alcohol consumers in the past year increased for most age groups, with a decline between 2002 and 2010 for those aged 12 to 18 (Table 3). Chileans between the ages of 19 and 34 had the highest prevalence of drinkers for most years, followed by those aged 35-44. These findings are based on selfreports of successive home surveys, using the same methodology and survey institution each year and with comparable large and high-quality samples.

High-risk drinking - drinking five or more drinks at one sitting at least once in the past month-was also part of the problem: the highest percentage was in the 19-25 age group (12-14\%), followed by those in the 26-34 age group (10-12\%). Figure 1 presents the percentage, by age, who reported drinking more than five drinks per occasion in the 
Figure 1

Prevalence of high risk drinking* during the last year among Chileans, by sex, age, socioeconomic status, 2008 and 2010

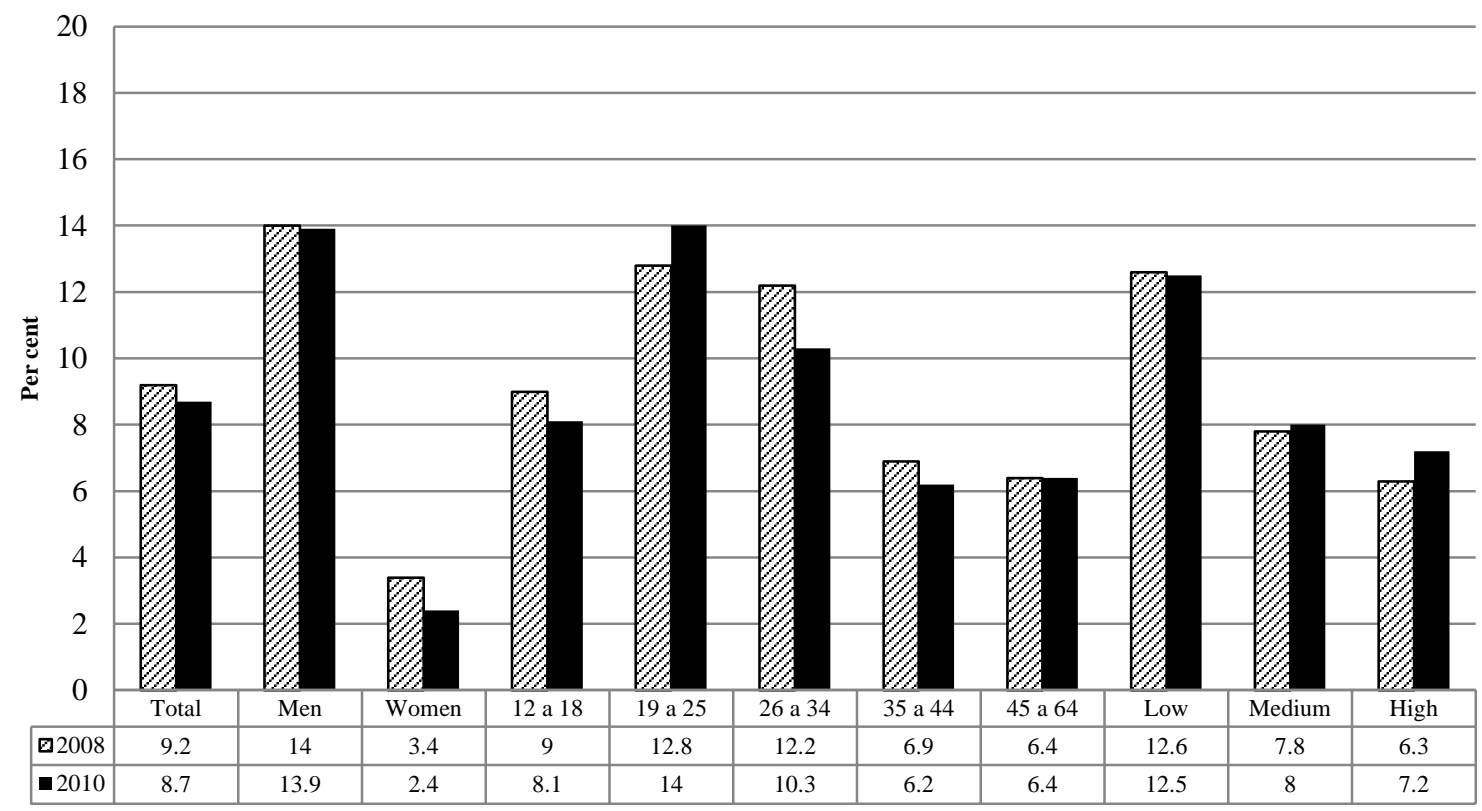

* High risk drinking: 5 or more drinks in one sitting at least once in the past month.

Source: Servicio Nacional para la Prevención y Rehabilitación del Consumo de Drogas y Alcohol, SENDA,

Ministerio del Interior y Seguridad Pública Chile. Observatorio Chileno de Drogas, SENDA

past year for 2008 and 2010. In Chile, there is a high prevalence of drinking in high-income sectors, but a higher prevalence of high-risk drinking among lower-income citizens. Information is not available about the number of high-income people seeking treatment, so these two groups cannot be compared on this dimension.

Although the trends in alcohol abuse according to DSM-IV criteria (American Psychiatric Association, 1994) were stable between 2002 and 2006, there were important differences within the population. The rate for males was about four times higher than females. And of the five age groups, which covered ages 12 to 64 , those aged 19-25 had the highest prevalence of this classification (25\%) (Consejo Nacional para el Control de Estupefacientes [CONACE], 2007).

According to a 2007 report, alcohol dependence was one of the five leading specific causes of burden of disease in Chile (Ministerio de Salud de Chile, 2007). Alcohol was a contributing cause in one of every 10 deaths and was the first leading risk factor associated to the highest burden in terms of disability-adjusted life years (DALY's). In the study based on the global burden of disease, Rehm et al. (2006) noted the following 2002 estimates for the subregion of the Americas that included Chile (AMR B): alcohol-attributable deaths 248,000, representing $9.2 \%$ of all deaths in the sub-region; alcohol-attributable disabilityadjusted life years (DALYs) 9,636,000, representing 11.8\% of all DALYs in the sub-region (Rehm et al., 2006; cited in Monteiro, 2007, Table 2.5).
A study of selected mortality rates where alcohol could be a contributor factor (Food and Agriculture Organization of the United Nations [FAO], 2003) identified a standardized mortality rate of 20.49 per 100,000 for liver cirrhosisalmost three and four times higher than in the United States and Canada respectively, and, in 2004, higher than most Latin American countries, with the exception of Mexico (see Table 4; also Alonso, Garmendia, Aquirre, \& Searle, 2010). While ischemic heart disease and cerebrovascular diseases had the highest mortality rates in Chile, the contribution of alcohol to these diseases was likely not as dominant as it is for cirrhosis. Alcohol's influence could be in interaction with tobacco and hypertension; together, these constituted the three leading burden risk factors for years lost due to premature mortality - that is, earlier than life expectancy.

An overview of automobile crashes and fatalities for 1997 to 2011 is provided in Figure 2. The number of car crashes and fatalities remained fairly steady, with small fluctuations, from 1997 to 2001, but car crashes began to increase in 2003 and have increased substantially in 2007 (Comisión Nacional de Seguridad de Tránsito [CONASET], 2007). Fatalities related to alcohol use and driving showed an abrupt increase between 2001 and 2003; after that, the levels tended to diminish slowly. To date, no reliable explanation has been found for these trends in traffic fatalities during 2001 and 2003, and from 2008 to 2011. The alcohol use law (2003) and increased fines on drinking and driving (2004) do not seem to have been a major factor, since the changes in rates occurred some 
years after these laws were passed. Only in 2012 (not shown) did the situation change substantially with strong positive outcomes.

\section{Table 4}

Standardized mortality rates (per 100 000) for liver cirrhosis, by WHO regional subgroupings (data shown is for most recent year available)

\begin{tabular}{lc}
\hline Country & Liver cirrhosis \\
\hline Ecuador & 15.45 \\
Argentina & 6.39 \\
Brazil & 11.31 \\
Chile & 20.49 \\
Colombia & 6.25 \\
Paraguay & 6.26 \\
Uruguay & 5.95 \\
Venezuela & 11.21 \\
Bahamas & 16.91 \\
Costa Rica & 7.81 \\
El Salvador & 12.41 \\
Mexico & 36.15 \\
Panama & 7.91 \\
Trinidad y Tobago & 9.55 \\
Cuba & 7.61 \\
\hline
\end{tabular}

SOURCE: World Health Organization. Department of Mental Health and Substance Abuse (2004). Global Status Report on Alcohol 2004. Singapore: WHO

\section{Alcohol Regulation in Chile}

Chile has a long history of legislation to regulate alcohol production, marketing, and consumption, as well as to control the effects of drinking on driving. When the National Alcohol Strategy was in development in 2009, the main piece of legislation governing these matters was Law 19,925-Sale and Consumption of Alcoholic Beverageswhich became effective in 2004 (Gobierno de Chile, 2004). According to this law, the density of authorized alcohol outlets is restricted to 1 per 600 inhabitants, although hotels and restaurants are not included in this restriction. The sale of alcohol is permitted seven days a week, while hours of sale are extended until 1:00 a.m. on weekdays, and to 3:00 a.m. on weekends, depending on the type of outlet.

Law 19,925 establishes that the minimum legal age to buy alcohol is 18, and forbids selling or serving alcohol to a minor in a bar, restaurant or package store. Selling or serving alcohol to someone who is obviously intoxicated is also prohibited. The law forbids drinking or being intoxicated in public places, and if a person is found drunk in a public place more than three times in the same year, the judge may order compulsory ambulatory medical or psychological treatment or involuntary hospitalization.

There have also been a number of regulations and laws pertaining to drinking and driving (e.g., Law 20068, under Transit, 2005). They apply to persons who drive vehicles or operate machinery while under the influence of alcohol, defined until 2012 as having a blood alcohol level of between 0.5 and one gram of alcohol per liter of blood, or while in a drunken state, defined as having one gram of alcohol or more per liter of blood. The sanctions and penalties can be administrative (such as suspension of the driver's license for varying periods, mandatory attendance of rehabilitation services, etc.), or may include fines or imprisonment, depending on the severity of the transgression.

Although Chilean laws on alcohol were often based on regulations that have shown some degree of effectiveness in developed countries, they apparently have failed to have an impact on the level of alcohol intake or on the consequences associated with it in Chile. The main explanation for this failure has been the low level of implementation of the existing legislation on alcohol, and low enforcement of regulations in place. As a country with a long tradition of producing wine, Chile has numerous cultural conventions associated with excessive drinking at different ages and among both sexes, and with high social tolerance for these behaviors. The policies surrounding alcohol are culturally influenced and constrained by public opinion.

For example, regulations permitting only one alcohol outlet license per 600 inhabitants, and limiting the hours of sale, are grossly undermined by other types of legal licenses and lack of rigorous police control; the prohibition of selling to minors is seldom enforced, and in most neighborhoods the population and the police turn a blind eye to the purchase of alcohol by minors. The acceptance of problem drinking may be explained by three factors: its pervasiveness; the fact that treatment interventions for intoxication in public places are only partially enforced, due to inadequacies in the records required to monitor the phenomenon over time; and (until very recently) infrequent police control of drinking and driving, owing to the limited resources traditionally allocated to the police for this purpose.

To date there have not been studies that link the 2004 law, mentioned above, with a decline in consumption among 12to 18-year-olds. However, it might be noted that during this time there were education initiatives, increased access to treatment among youth, and a high level of public alarm about drinking and related problems among youth. 
Figure 2

Car crashes and fatalities related to alcohol in Chile (1997-2011)

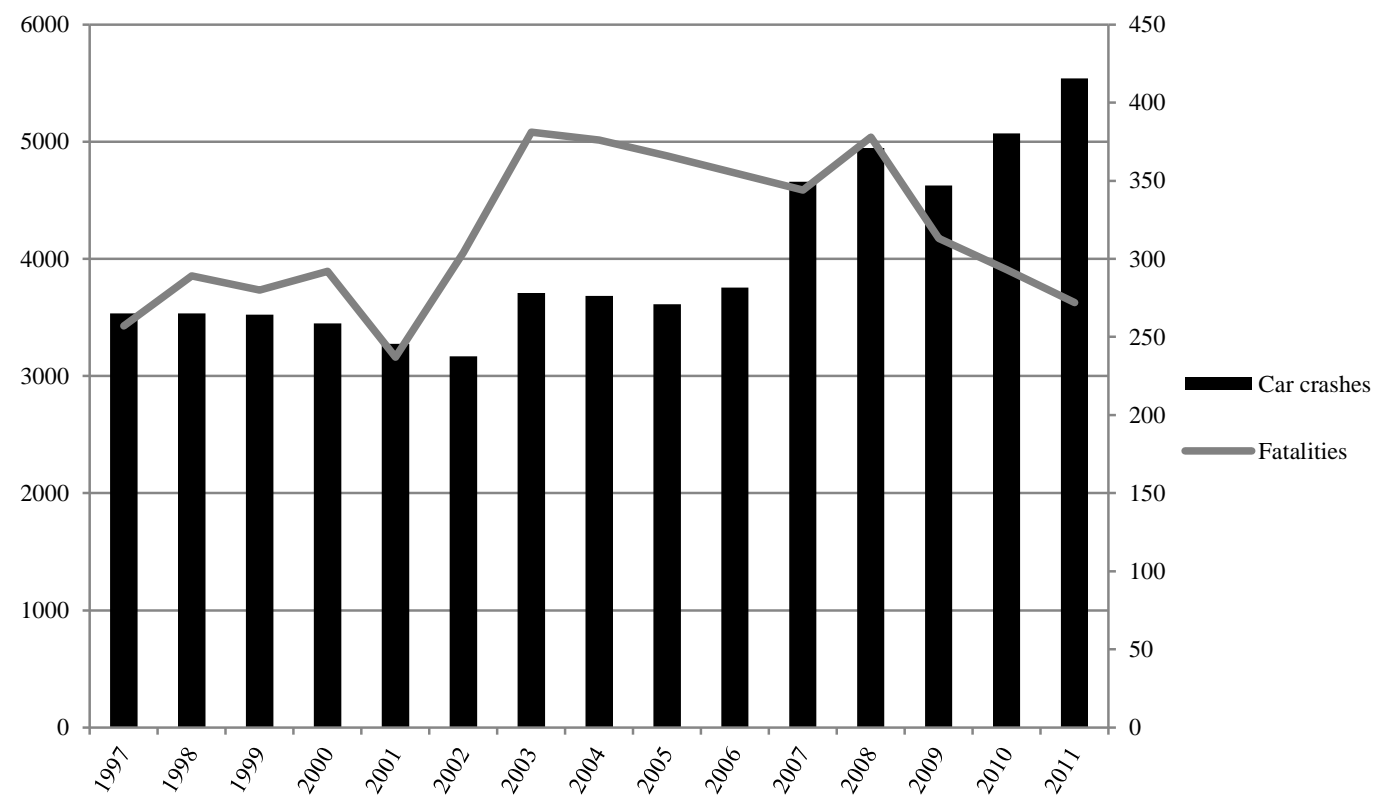

SOURCE: Comisión Nacional de Seguridad de Tránsito (CONASET). Siniestros de tránsito y consecuencias según causa (2000-2011). Available on: http://www.conaset.cl/conaset_web/contenido.php?id=73

Secretaría Ejecutiva. Comisión Nacional de Seguridad de Tránsito (2007). Siniestros de tránsito ocurridos en Chile y asociados a la presencia de alcohol en los participantes- Año 2007. Disponible en: www.conaset.cl

\section{Public Health Approach to Alcohol Problems}

Chile formulated its first national public health program on alcohol in 1957. This program was implemented in the recently created National Health Service, which was developing an extensive publicly funded network of primary, secondary and tertiary facilities. Many primary care centers implemented local programs for alcohol dependence management, with the participation of general practitioners, social workers and mutual-help rehabilitation groups. Since 1991, several initiatives have been developed by the Ministry of Health in the public system to promote screening and early intervention with problem drinkers, as well as to reinforce treatment and rehabilitation for alcohol and illicit drug abuse and dependence. A locally developed screening test, known by its Spanish acronym EBBA (Escala Breve de Beber Anormal) (Orpinas et al., 1991), was introduced nationally in primary health care (PHC) in the early nineties, and allowed earlier detection of people at risk, although it lacked a strong system of intervention to reduce risk.

In 2005, a guideline on preventive and therapeutic interventions, to be delivered by PHC workers, was introduced, as well as the Alcohol Use Identification Test (AUDIT), which replaced the EBBA. Nevertheless, the rate of detection and treatment of potential problem drinkers seeking health care in PHC remains very low, probably because the budget to support its application and the interventions is low. General practitioners and the PHC teams are overburdened with many patients and many other healthcare priorities.
The most successful mental health program in PHC, the treatment of depression, was started with new funding allocated to PHC for the treatment of each person admitted to the program. This made it possible to hire additional professionals and to have an adequate supply of medication.

Two new developments were introduced in this period. Firstly, in 2006 a program was initiated providing a route of referral from justice to healthcare for people who had committed violations of the law at a non-criminal level (drinking and driving, neighborhood disturbance, family violence), as a complementary or alternative measure to sanctions (Ministerio de Salud de Chile, 2006). Secondly, in 2007 the treatment of adolescents with alcohol and drug disorders was included in the Program of Explicit Health Guarantees. According to this program, both the public health care system and the private health insurance industry must offer an explicit set of guarantees in terms of access, quality, opportunity, and financial coverage for 69 priority diseases. Details of the guarantees are clearly specified for each disease, including the populations entitled to receive them, types of interventions, maximum waiting time, minimum qualifications of providers, and ceilings for copayments. Nevertheless, the actual demand for the treatment of alcohol and drug disorders remains low. A possible reason for this is that the budget allocated to this program includes only modest ambulatory treatment for people under 20 years and with mild substance use disorders and fails in providing outreach programs for 
active detection or intensive ambulatory, hospital or residential care.

The AUDIT (Babor, Higgins-Biddle, Saunders, \& Monteiro, 2001; Saunders, Aasland, Babor, De La Fuente, \& Grant, 1993) has also been used since 2006 in the Preventive Health Examination (Examen de Medicina Preventiva, n.d.) (EMP). This is a screening test for seven pathologies, including hypertension and diabetes, which is provided on a voluntary basis to every person entering the public and private health system, and by sanitary protocol to young people, those over 60, pregnant women (since 2009), and adults in general at the PHC level. No records of clinical outcomes of the EMP are available.

A new development, since 2011, is a program of brief interventions at the PHC level, using AUDIT as screening tool, covering 23 counties in the Metropolitan Region but expanding on a progressive and national basis. Preliminary analysis of the data indicate that among the 55,422 persons who have been screened, including 24,051 men and 31,371 women, $16.3 \%$ scored between 8 and 15 on the AUDIT, including $29.3 \%$ of the men and $6.4 \%$ of the women. These statistics under-report the total number of registrants, since the number of people reached in 2012 was nearly 200,000 .

\section{Steps Toward a National Strategy}

\section{Principal developments 2008-2009}

Noteworthy contextual developments were the high rate of alcohol-related trauma, including drinking and driving; the high rate of liver cirrhosis mortality and morbidity; the high rate of heavy drinking, including among youth and young adults; and gender differences. Even when there were a variety of laws in place and several public health initiatives, the level of implementation of some potentially effective interventions was considered to be low or even nonexistent. These weak outcomes reinforced the need for more effective action. With this perspective, during the period from 2008 onward, a growing number of public health professionals, including a few in the Ministry of Health, became aware of the opportunity for a comprehensive proposal for a public policy on alcohol for Chile. The Mental Health Department in the Ministry of Health became aware of the recently published new evidence on effectiveness of public policies (Babor et al., 2003) and the progress at the WHO/PAHO on this issue, which contributed toward planning toward a national strategy. This attracted greater attention to the goal of having a national and comprehensive strategy on alcohol, with a public health perspective. One of the steps was to ask for advice from CAMH, within the framework of an agreement

Table 5

Key Steps toward a National Alcohol Strategy: A Nonlinear Process

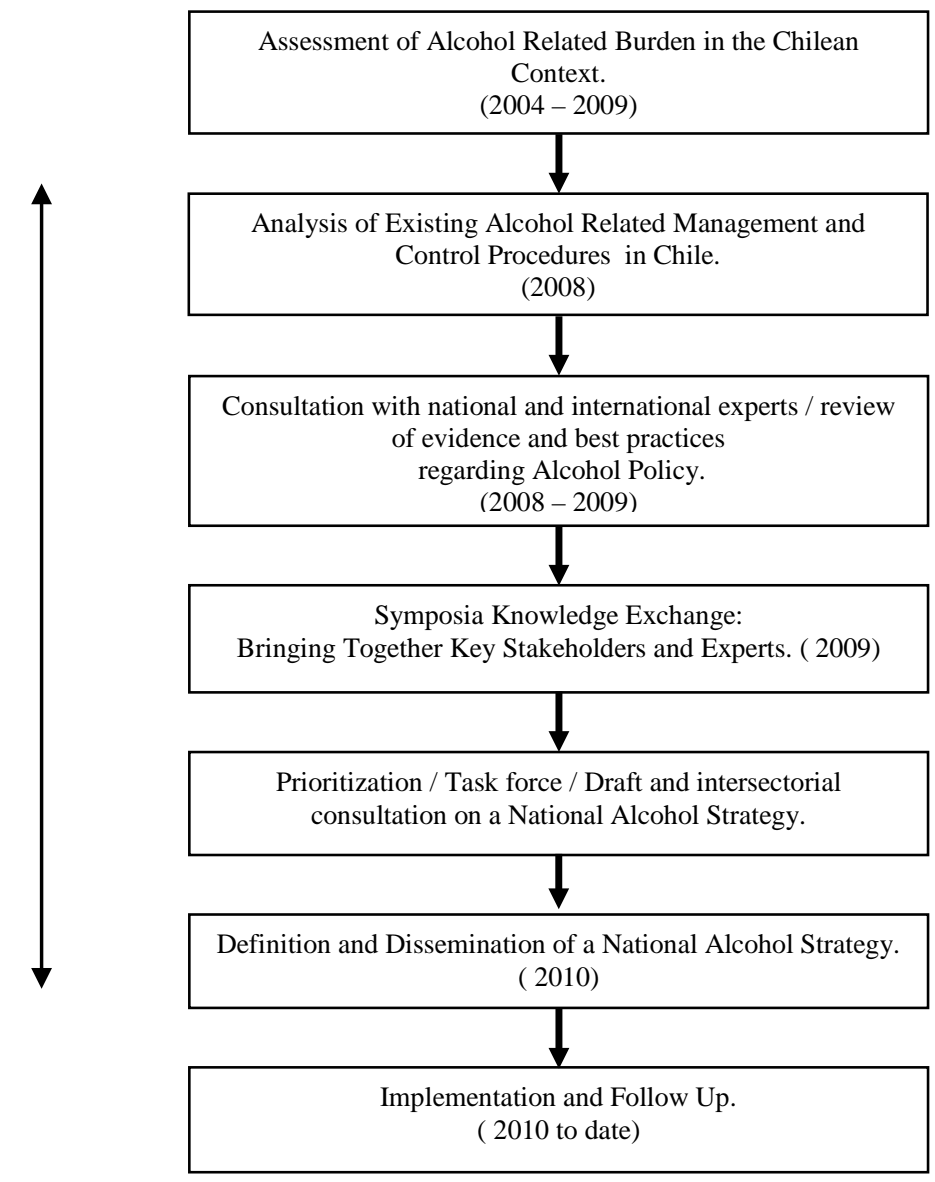


of collaboration signed three years earlier. The seminal report delivered by staff at CAMH (Giesbrecht, Marquez, \& Sapag, 2009) was a useful resource from which the definitive document could be articulated. The key steps of the policy planning and refinement process are outlined in Table 5.

\section{Principles}

The following points oriented the preparatory phase of the Chilean strategy:

a. Alcohol problems are substantial and costly, and there is an urgent need to address them.

b. Alcohol policy should be linked with public health policy and be a part of it.

c. The country's alcohol consumption rate, as well as the related problems, may be modified with public strategies.

d. Research conclusions support the evidence-based efficacy and cost efficacy of public policies that are amenable to being implemented by governments. e. The main measures need to reach the whole population, not only focus on the heavy drinkers.

f. Central components of a policy on alcohol are rational use of alcoholic beverages, limits to its availability, taxes, preventive measures on drinking and driving, and investments on screening and treatment, particularly in PHC. Massive education campaigns may be useful, but only as a complement to, and a support of, more effective policy measures.

g. All effective alcohol policy measures have important implications outside the management of the health sector, and need a quota of persistent, dedicated and informed professionals. It is likely that public opinion will be opposed to some initiatives to reduce access to alcoholic beverages, and the alcohol production and retailing industries are expected to resist any enhanced regulatory measures.

\section{Table 6}

\section{Recommendations for a national strategy on alcohol and public health ${ }^{1}$}

\section{Social determinants of health}

1.1 Examine the variations in the social determinants of health as they relate to alcohol in order to explore differences in access to alcohol, high risk drinking and access to social and health services for those who are high risk drinkers or dependent on alcohol.

1.2 Consider alcohol policies within the context of a comprehensive health approach and connections with other policies, which can encourage access to other prevention policies, services, treatment among high-risk drinkers and persons with multiple health risks.

1.3 Assess the challenges of implementing, and potential impact of a national alcohol strategy, on the indigenous people of Chile.

2. Persuasion, Information Dissemination and Marketing

2.1 The focus of information campaigns should be on promoting a more accurate understanding of the social and cultural contexts of alcohol use, alcohol industries, marketing of alcohol, responsibility of policy makers, opportunities and responsibilities of citizens, and demonstrating/illustrating the potency of population level alcohol control strategies.

2.2 Develop and implement as a complimentary component of more effective interventions and designed to support these campaigns.

2.3 Review current standards and controls on alcohol marketing, advertising and promotion to assess their enforcement and impact. In line with the precautionary principle, undertaken modifications in order to reduce the potential of alcohol advertising stimulating higher rates of overall alcohol use, high-risk behaviour and encouraging drinking among under-age youth and high-risk drinkers.

\section{Availability of Alcohol}

3.1 Assess, the current regulations about density of alcohol outlets in Chile with the aim of setting a. lower density threshold - fewer outlets per capita -- and monitoring to assess its impact.

3.2 Asses current selling practices involving hours of sale to determine if early opening or late closing hours are associated with high risk drinking. Pilot an intervention which examines the impact of reducing hours of sale on alcohol-related social and health problems.

3.3 Determine how concentration and 'mix' of outlets contribute to selling price, serving practices, intoxication and drinking-related disruptions and violence in those areas with high concentration. Implement a pilot project to reduce alcohol-related problems by providing enforceable guidelines on the mix of outlets and number per city block.

3.4 Assess the current minimum legal drinking age (MLDA) and current levels of enforcement to determine if higher legal age and enhanced enforcement would reduce high-risk drinking among youth.

\section{Alcohol Taxes and Retail Prices}

4.1 Establish a retail floor price, or minimum price, for each beverage group - based on the amount of pure ethanol in typical size containers, with comparable arrangements for 'on premise' sales.

4.2 For similar beverage volume, the retail floor prices for alcohol should be substantially higher than non-alcohol beverages - for example, $500 \mathrm{ml}$ of beer should cost substantially more than $500 \mathrm{ml}$ of orange juice.

4.3 Systematically review current tax and price policies with the aim to implement policies that insure that cost of the lowest priced and most prevalently sold beverages at least keep pace with the consumer price index, with indexing arrangements to insure that they exceed the Consumer Price Index.

4.4 Review options for differential pricing by beverage strength. The base price should be for the weakest strength beverage and prices should be set higher for those beverages that have more ethanol per volume.

\section{Alcohol Service and Retailing Policies}

5.1 Conduct an unobtrusive evaluation of current serving programs and guidelines to determine if they are associated with lower rates of overserving, service to minors or on-site drinking-related problems and disruptions in the premises.

5.2 Implement the most effective server training programs and bar policy programs, including violence reduction programs, and monitor and assess their impact on heavy drinking and alcohol-related damage. 


\section{Drinking and Driving Prevention Policies}

6.1 Conduct a review of current drinking and driving prevention policies and legislation in Chile, and examine: (a) their expected potency in reducing rates of drinking and driving; and (b) current level of enforcement.

6.2 Develop an action plan to facilitate enhanced enforcement for those current policies already in place and considered to have a high potential for impact,

6.3 Develop and implement a pilot project of policies identified in the literature having high potential, but currently not in place in Chile, and track their impact on drinking and driving rates and alcohol-related crashes.

6.4 Provide bi-annual reports, with data/information disaggregated by region of Chile, on the status of policies and programs, level of enforcement, and trends in drinking and driving rates, incidents, alcohol-related crashes, and mortality rates.

6.5 Develop, implement and monitor special campaigns to reducing drinking and driving incidents, crashes and deaths during special times of the week (weekends) or day (evening or early morning) when incidents are most prevalent, including national holidays. These campaigns should include increased enforcement including periodic randomized roadside checkpoint campaigns.

6.6 Examine the current legal blood alcohol concentration legislation, and explore the benefits and challenges of setting a lower BAC level e.g., as is common in many European countries.

\section{Screening and Brief Interventions for High-Risk Drinkers}

7.1 Develop, implement and evaluate several small-scale pilot studies that provide a screening and brief intervention for high-risk drinkers. The four proposed settings include: a university-based clinic, an emergency room service, a regular medical clinic, and a work-place setting.

7.2 Develop a pilot web-based service that will allow members of the general public to assess their drinking levels and patterns, obtain guidance for monitoring their consumption and tracking their initiatives to reduce high-risk drinking.

7.3 Explore options for linking the provision of brief intervention with treatment services for chronic conditions or trauma that may have an underlying alcohol component.

7.4 Develop a pilot project for offering a brief intervention for high-risk drinkers among the indigenous populations.

\section{Community Action and Harm Reduction}

8.1 Facilitate the development of several demonstration projects that focus on the community settings and include alcohol policy/regulatory interventions as a central component and include a combination of evidence-based policies and prevention strategies, such as, local controls on density of outlets or hours of sale, increased server training and monitoring - to reduce access to under-age youth or to intoxicated patrons and customers, increased enforcement of drinking and driving, and controls on local advertising and alcohol promotion.

8.2 Supplementary components of a community-based demonstration project might include specialized, evidence-based, and viability programs focusing on youth, parents, workers, or pregnant women, within the resources provided.

\section{Training and Capacity-Building}

9.1 Implement an on-site training and capacity building course especially for applicants who will have a central role in implementing the dimensions of the National Alcohol Strategy. This one-week course would be an overview course focusing on all of the key dimensions of the National Alcohol Strategy (see recommendations in this section [I]). It would focus on the goals, rationale, supporting international research, and the relevance of the recommended dimensions to national and local alcohol-related problems

9.2 Depending on outcome of this pilot course, a few follow-up courses should be offered which provide more detailed training and capacity building. Each course might focus on 2 to 3 dimensions of the National Alcohol Strategy.

9.3 Develop a media/information campaign on the key dimensions of the National Alcohol Strategy.

\section{Monitoring, Evaluation and Follow-up}

10.1 In order to fine-tune the key components of an alcohol strategy and provide useful baseline data,, develop a synthesized overview, of the current status of alcohol use, drinking patterns, problems and policy enforcement -- built on existing data (official statistics, surveys, published reports), supplemented with new data as required.

10.2 For each policy strategy listed above ( 1 to 9$)$ develop a monitoring protocol that facilitates tracking of: the implementation process, challenges encountered, resource allocations and level and type of enforcement. This protocol should also document changes in relevant laws and regulations. It should also track the month/year of the introduction of major prevention campaigns or major changes in the focus and scope of existing campaigns.

10.3 An evaluation protocol should be developed for policy dimensions listed above.

10.4 On a periodic basis a debriefing/follow-up report should be prepared on each policy.

${ }^{1}$ Based on Giesbrecht, Marquez, \& Sapag, 2009

\section{Recommendations}

The recommendations in Table 6 are organized into 10 groups (based on Giesbrecht et al., 2009): social determinants of health; persuasion, information dissemination, and marketing; availability of alcohol; alcohol taxes and retail prices; alcohol service and retailing policies; drinking and driving prevention policies; screening and brief interventions for high-risk drinkers; community action and harm reduction; training and capacity-building; and monitoring evaluation and followup. There is substantial convergence between the list of building blocks recommended by Monteiro (2007, p. 36) and these 10 groups. The 10 groups of recommendations also overlap with the 10 policy options (noted earlier) provided in the WHO discussion paper (WHO, 2009), and evidence-based interventions outlined by Babor et al. (2003) and subsequently in Babor et al. (2010). The recommendations in Table 6 are summarized from Giesbrecht et al. (2009). (Please refer to that document for a full statement and rationale.)

The recommendations provided by the CAMH advisors were fully included in the definitive document and provided a sure route for the ongoing exchange of opinions 
with, and decisions taken by, the health authorities, as well as in the advocacy efforts with other sectors of government.

\section{Deliberations and Planning}

Different ministries were involved in responding to this issue; they overcame these contextual challenges to coordinate a multi-sectorial national effort for developing a comprehensive response. By 2009, a number of sectors of the national government, including the Ministries of Economy and Finance, Transportation, Labor, Interior, Education, and Health, were involved in the process of developing a national strategy on alcohol.

Two international seminars held in successive years, 2009 and 2010, provided opportunities for reviewing, confirming, and building the core ideas of the national policy document in preparation, and allowed a wide range of stakeholders to enhance their knowledge of the policies and offer their support. Officials from different sectorsacademics, civil society and even the alcohol industrywere participants.

Between the first and the second seminar, two groups were formed. A small technical commission (eight people, mainly from the health sector) was created: they developed successive drafts on discussions and reviews of the relevant literature. A second group, composed of official representatives of the different ministries, reviewed and discussed each of the chapter drafts. During the second seminar, the audience was able to receive new data from the national health survey conducted during 2009 (Ministerio de Salud de Chile, 2010a) and a new national study on alcohol use (CONACE, 2010), which confirmed the urgent need for the strategy being developed and the existence of opportunities to implement it. Finally, a public formal consultation made available on the web page of the Ministry of Health finished the elaboration process of the document.

The National Strategy on Alcohol was passed in March 2010 (Ministerio de Salud de Chile, 2010a), two months before the WHO Health Assembly and Resolution on Global Strategy. A foreword was drafted by two ministers, from different government coalitions, during the transitional period. The document had the following purposes: (i) To be a source of evidence-based information for public policies, involving different sectors, in order to initiate the common goal of changing the social culture of Chileans' use of alcohol over the next few years; (ii) To emphasize the need for an approach involving simultaneous measures in a synergistic and coordinated approach; and (iii) To gain the support of a strong and durable political and technical will that includes evaluation of the strategy.

To summarize, the policy document included the following nine lines of action:

o Public information on alcohol and control of alcohol advertising

o Control of alcohol availability

o Alcohol taxes and retail sale prices to control high-risk drinking and alcohol-related harm o Precautionary policies on alcohol distribution and retail sales

o Interventions to control traffic accidents and harmful alcohol consumption

o Strategies to reduce hazardous alcohol consumption and provide enhanced health care

o Community action and local government initiatives

o Education, personnel and job training

o Monitoring and evaluation

\section{Discussion}

This article summarizes the steps toward the development of a National Alcohol Strategy in Chile. The country approached the challenge using a proactive and multi-step process. It included a systematic assessment of the alcohol public health problem, as well as of the existing regulations to address it. It also involved national and international experts and the participation of different relevant stakeholders. Chile became one of the first countries in Latin America to have an explicit and comprehensive National Alcohol Strategy.

The Global Status Report on Alcohol and Health for the Americas (WHO, 2011) provides cross-sectional status information on several policy dimensions, including excise taxes on beverages, minimum legal drinking age for onpremise and off-premise venues, restrictions on hours and days of sale, maximum legal blood alcohol concentration when driving, and legally binding regulations on alcohol advertising, product placement, sponsorship, and sales promotion. This is a rich resource for an overview comparison of multiple countries.

The National Strategy included nine lines of action (Ministerio de Salud de Chile, 2010b), described above, that apply to a wide variety of aspects such as pricing policies, retail sales practices, information for the public, alcohol marketing, education, effective action regarding drinking and driving, early and adequate detection, and treatment of those affected by hazardous use. It also considers civil participation - that is, from non-government agencies and organizations - and the roles that local, municipal and regional authorities may play in the National Alcohol Strategy.

The powerful alcohol industry in Chile maintains a wide presence via sponsorship and publicity activities, and has developed highly visible programs under the "enterprise social responsibility" umbrella, focusing on initiativessuch as educative interventions in schools and designateddriver spots-where the evidence of harm-reduction impact is limited or non-existent (Anderson et al., 2009; Babor et al., 2010; Giesbrecht, 2007). Public opinion is frequently well informed and impressed by the wine quality international awards, the magnitude of exports, and the industry's revenues and profits. The plan for the national alcohol strategy was to build a powerful document, and then to influence public opinion with a new basic message: Now we have powerful tools for creating good outcomes, beyond the usual claims, denunciations or complaints. 
Since 1990, the Ministry of Interior through CONACE had been in charge of the national strategy on drugs, and the policies for alcohol were the responsibility of the Ministry of Health. The Servicio Nacional para la Prevención y Rehabilitación del Consumo de Drogas y Alcohol (SENDA) (National Service for the Prevention and Rehabilitation of Drug and Alcohol Use, 2011), was created in October 2011, and included both illicit drugs and alcohol among its functions. SENDA developed the 20112014 National Strategy on Drugs and Alcohol, which incorporated several of the recommendations of the National Strategy for Alcohol elaborated at the Ministry of Health in 2010 (CONACE, 2011). That strategy is aimed at achieving (1) a $15 \%$ reduction in the annual levels of alcohol consumption among children of school age, (2) a $15 \%$ reduction in dangerous levels of alcohol consumption among the adult population, and (3) a $20 \%$ reduction in road traffic accidents related to alcohol consumption among the general population. In January 2012, Congress passed an amendment to the traffic law, decreasing the levels of blood alcohol required for a person to be legally considered "under the influence of alcohol" and "drunk," increasing resources to measure alcohol in the breath, and initiating a national program to control motor vehicle drivers (González-Wilhelm, Johnson, Carnevali, \& Ruiz, 2012). Particularly outstanding has been the enforcement of the new law by the police, and an extensive education campaign.

In addition, the National Health Strategy (ENS) (Ministerio de Salud de Chile, 2011), launched in December 2011, includes two specific goals for 2020: a $10 \%$ reduction of the alcohol consumption rate in young people between 15 and 24 years old, and a $10 \%$ reduction in disability associated with selected and severe mental health disorders, including alcohol dependence and harmful use. Many other goals in the ENS- such as those for chronic diseases, casualties and injuries, and child and adolescent healthare also indirectly related to alcohol use. They represent further opportunities for synergistic efforts in health policy planning and development.

It seems that an additional task force is needed, besides the overloaded Health Public Sector. Unfortunately, the widely shared understanding among health professionals of the challenges and benefits of alcohol policies is not as prevalent among government officials, academics or other parties.

Some initiatives remain in the pipeline. It is hoped that data from a study of the avoidable costs of alcohol will provide powerful support for unavoidable economic policy decisions. It is an open field for collaboration with other countries-in this case, with Canada, through CAMH. Some changes in the legislative field have not been achieved, but are still available as important options, such as restrictions on the promotion of alcohol and drinking in the media.

The issue of alcohol tax increases has surfaced in political agendas from time to time, just as a health measure, without any support from other sectors, or as a measure for growing the general budget, to allow investment in highquality education. However, none of these initiatives have yet reached the legislative stage. There has not been complete success in convincing ministers of health and middle managers in different sectors to advocate at the government level for the use of alcohol taxes as a public health measure. It has been challenging to build support in Parliament (Legislative Congress) for increasing alcohol taxes, establishing alcohol retail floor prices, and implementing strong warning labels for alcohol bottles, among other important measures. Chilean culture is very permissive of excessive drinking for all ages, and drinking alcoholic beverages is part of the Chilean identity. Consequently, to increase taxes would be a very unpopular policy for any government.

The Chilean example provides many positive lessons in how a country can connect international evidence with its local needs to create a powerful process to address a public health issue. The process followed key principles for excellence in policy development. In particular, it emphasized the importance of building an inter-sector response through an open and focused knowledge exchange and prioritization of action steps.

Leadership is, of course, a key ingredient for success in alcohol policy development (Jernigan, Monteiro, Room, \& Saxena, 2000). Building a national alcohol strategy requires the participation of many actors and the analysis of different interests in order to arrive at a public-healthoriented policy. Senior officials at the Ministry of Health played a strong role during the process, and this was critical for motivating other sectors and key players, such as labor, education and economy. The WHO's global strategy, and the regional coordination that is one of its follow-up mechanisms, was a useful tool for homogenizing progress between countries and for sharing respective strategies and experiences.

Alcohol policy initiatives are being implemented in Latin America (Babor \& Caetano, 2005; Medina-Mora, 2007; Monteiro, 2007). Many of them have been focused on specific areas to prevent alcohol-related harm; for example, by tackling alcohol drinking and driving, or defining legal minimum age for on-premise sales of alcoholic beverages (Andreuccetti et al., 2011; Pelaez Mariscal \& da Silva, 2010; Sanchez et al., 2011; Stewart, Silcock, \& Wegman, 2012). Currently, there is a growing recognition of the urgent need for the development of comprehensive national alcohol policies (Babor et al., 2012; Fiestas, 2012; Matos de Andrade, 2011). The experience of Chile may be relevant to inform those processes of alcohol policy development and implementation in the region and beyond.

\section{Acknowledgements}

The authors acknowledge financial support from the following organizations: Centre for Addiction and Mental, Toronto, Ontario, Canada; Mental Health Department, Chilean Ministry of Health, Santiago, Chile; and School of Public Health, University of Chile, Santiago, Chile. The 
views and opinions are those of the authors and do not necessarily reflect the policies of the organizations acknowledged.

\section{References}

Alonso, F. T., Garmendia M. L., Aquirre, M. D., \& Searle, J. (2010). Mortality trend from liver cirrhosis in Chile from 1990 to 2007. Revista Médica de Chile, 138, 1253-1258.

American Psychiatric Association. (1994) Diagnostic and Statistical Manual of Mental Disorders (DSM-IV) (4th ed.). Washington, DC, United States: Author.

Anderson, P., Chisholm, D., \& Fuhr, D. (2009). Effectiveness and cost effectiveness of policies and programmes to reduce the harm caused by alcohol. Lancet, 373, 2234-2246.

Andreuccetti, G., Carvalho H. B., Cherpitel C. J., Yu Y., Ponce J. C., Kahn T., \& Leyton, V. (2011). Reducing the legal blood alcohol concentration limit for driving in developing countries: a time for change? Results and implications derived from a time-series analysis (2001-10) conducted in Brazil. Addiction, 106, 21242131.

Babor, T., Caetano, R., Casswell, S., Edwards, G., Giesbrecht, N., Graham, K., \& Rossow, R. (2003). Alcohol, no ordinary commodity: Research and public policy. Oxford, United Kingdom: Oxford University Press.

Babor, T., Caetano, R., Casswell, S., Edwards, G., Giesbrecht, N., Graham, K., \& Rossow, I. (2010). Alcohol: No ordinary commodity - Research and public policy (2nd ed.). Oxford, United Kingdom: Oxford University Press.

Babor, T., Rehm, J., Jernigan, D., Vaeth, P., Monteiro, M., \& Lehman H. (2012). Alcohol, diabetes, and public health in the Americas. Revista Panamericana de Salud Pública, 32, 151-155.

Babor, T. F., \& Caetano, R. (2005). Evidence-based alcohol policy in the Americas: strengths, weaknesses, and future challenges. Pan American Journal of Public Health, 18, 327-337.

Babor, T. F., Higgins-Biddle, J. C., Saunders, J., \& Monteiro, M. G. (2001). AUDIT, the Alcohol Use Disorders Identification Test: Guidelines for use in primary health care (2nd ed.). Geneva, Switzerland: World Health Organization.

Brand, D., Saisana, M., Rynn, L., Pennoni, F., \& Lowenfels, A. (2007). Comparative analysis of alcohol control policies in 30 countries. PLOS Medicine, 4, 0752-0759.

Comisión Nacional de Seguridad de Tránsito. Gobierno de Chile (CONASET). (2007). Siniestros de tránsito ocurridos en Chile y asociados a la presencia de alcohol en los participantes [Traffic accidents occurred in Chile and associated with the presence of alcohol in participants]. Retrieved from http://www.conaset.cl/ cms_conaset /archivos/Alcohol\%20total\%202007.pdf

Consejo Nacional para el Control de Estupefacientes (CONACE) (2007). Séptimo estudio nacional de consumo de drogas, 2006 [Seventh national survey on drug use, 2006]. Retrieved from http://antiguo. conacedrogas.cl/portal/images/Pdf/7oEstudioNacional ConsumoDrogasChile_Conace2006.pdf

Consejo Nacional para el Control de Estupefacientes (CONACE) (2010). Noveno Estudio Nacional de Drogas en Población General de Chile, 2010 (9th National Drug Study in General Population, 2010). Retrieved from: http://www.senda.gob.cl/wpcontent/uploads/2011/06/novenoestudionacional2010. pdf

Consejo Nacional para el Control de Estupefacientes (CONACE) (2011). Estrategia nacional de drogas y alcohol 2011-2014 [2011-2014 National strategy on drugs and alcohol]. Retrieved from http://www. senda.gob.cl/wp-content/uploads/2011/05/ estrategia_drogas_alcohol_2011_20141.pdf

Fiestas, F. (2012). Reducing the burden of diseases caused by alcohol use in Peru: Evidence-based approaches. Revista Peruana de Medicina Experimental y Salud Pública, 29, 112-118.

Food and Agriculture Organization of the United Nations (FAO). (2003). World drink trends 2003. Cited on: Department of Mental Health and Substance Abuse. World Health Organization (2004). Global Status Report on Alcohol 2004. Singapore: WHO. Retrieved from http://www.who.int/globalatlas/default.asp

Giesbrecht, N. (2007). Reducing alcohol-related damage in populations: Rethinking the roles of education and persuasion interventions. Addiction, 101, 1345-1349.

Giesbrecht, N., Marquez, J., \& Sapag, J. C. (2009). A National alcohol strategy for reducing high-risk drinking and damage from Alcohol in Chile. Toronto, Canada: Centre for Addiction and Mental Health.

Giesbrecht, N., Stockwell, T., Kendall, P, Strang, R., \& Thomas, G. (2011). Alcohol in Canada: Reducing the toll through focused interventions and public health policies. Canadian Medical Association Journal, 183, 450-455.

Gobierno de Chile. (2004). Ley Número 19.925 Expendio y Consumo de Bebidas Alcohólicas. Congreso Nacional de Chile. Retrieved from http://www.leychile.cl/N?i= 220208\&f=2012-09-14\&p=

Gobierno de Chile. Comisión Nacional de Seguridad de Tránsito. (2009). Siniestros de tránsito ocurridos en Chile asociados a la presencia de alcohol en conductores, pasajeros o peatones año 2009 (Traffic accidents occurred in Chile associated with the presence of alcohol in drivers, passengers or pedestrians in 2009). Retrieved from http://www. sigweb. cl/biblioteca/AccidentesPorAlcohol.pdf

González-Wilhelm, L., Johnson, J., Carnevali, R., \& Ruiz, G. (2012). Alcohol zero tolerance law in Chile: strengths, flaws and shortcomings that should not be overlooked]. Revista Médica de Chile, 140, 939-945.

Hospedales, J., Barcelo, A., Luciani, S., Legetic, B., Ordunez, P., \& Blanco, A. (2012). NCD prevention and control in Latin America and the Caribbean: A regional approach to policy and program development. Global Heart, 7, 73-81.

Jernigan, D. H., Monteiro, M., Room, R., \& Saxena, S. (2000). Towards a global alcohol policy: alcohol, 
public health and the role of WHO. Bulletin of the World Health Organization, 78, 491-499.

Matos de Andrade, T. (2011). Reflections on drug policies in Brazil. Ciência \& Saúde Coletiva, 16, 4665-4674.

Medina-Mora, M. E. (2007). Mexicans and alcohol: patterns, problems and policies. Addiction, 102, 10411045.

Ministerio de Salud de Chile. (2006). Programa de atención y rehabilitación de personas con beber problema y sanciones por la Ley de Alcoholes [Care and rehabilitation program for people with drinking problems and penalties for Alcohol Law]. Santiago, Chile: MINSAL.

Ministerio de Salud de Chile. (2007). Departamento de Epidemiología. P. Universidad Católica de Chile. Segundo estudio de carga de enfermedad y carga atribuible, Chile 2007 [Second study of attributable burden of disease, Chile 2007]. Santiago, Chile: MINSAL.

Ministerio de Salud de Chile. (2010a). Encuesta nacional de salud 2009-2010. Retrieved from http://www.redsalud.gov.cl/portal/url/item/99bbf09a9 08d3eb8e04001011f014b49.pdf

Ministerio de Salud de Chile. (2010b). National alcohol strategy: Reduction of high-risk alcohol consumption and its social and health consequences. Retrieved from http://www.minsal.gob.cl/portal/url/item/9bb28 7857186de00e04001011f011a4a.pdf

Ministerio de Salud de Chile. (2011). Estrategia nacional de salud 2011-2020. Retrieved from http://www.minsal. gob.cl/portal/url/item/b89e911085a830ace040010165 0115af.pdf

Monteiro, M. G. (2007). Alcohol and public health in the Americas: a case for action. Washington, DC, United States: Pan American Health Organization.

Orpinas, P., Valdés, M., Pemjeam, V., Florenzano, R., Nogueira, R., \& Hernández, J. (1991). Validación de una escala breve para la detecciónde beber anormal (EBBA) [Validation of a brief scale to detect abnormal drinking (EBBA)]. In R. Florenzano, N. Horwitz, M. Penna, \& M. Correa (Eds.), Temas de salud mental y atención primaria de salud [Issues of mental health and primary health care] (pp. 185-193). Santiago, Chile: Corporación de Promoción Universitaria.

Pelaez Mariscal, I. M., \& da Silva, E. C. (2010). Accidentes de tránsito y el consumo de alcohol en una unidad de urgencia de La Paz, Bolivia [Traffic accidents and use of alcohol in an emergency unit in La Paz, Bolivia]. Revista Latino-Americana de Enfermagem, 18, 613619.

Rehm, J., \& Monteiro, M. (2005). Alcohol consumption and burden of disease in the Americas: implications for alcohol policy. Pan American Journal of Public Health, 18, 241-248.

Rehm J., Patra, J., Baliunas, D., Popova S., Roerecke, M., \& Taylor, B. (2006). Alcohol consumption and the global burden of disease, 2002. Geneva, Switzerland: WHO Department of Mental Health and Substance Abuse, Management of Substance Abuse.

Sanchez, I. I., Villaveces, A., Krafty, R. T., Park, T., Weiss, H. B., Fabio, A., \&Gutierrez, M. (2011). Policies for alcohol restriction and their association with interpersonal violence: A time-series analysis of homicides in Cali, Colombia. International Journal of Epidemiology, 40, 1037-1046.

Saunders, J. B., Aasland, O. G., Babor, T. F., De La Fuente, J. R., \& Grant, M. (1993). Development of the Alcohol Use Identification Test (AUDIT): WHO collaborative project on early detention of persons with harmful alcohol consumption-II. Addiction, 88, 791-804.

Servicio Nacional para la Prevención y Rehabilitación del Consumo de Drogas y Alcohol. (2011). SENDA, Ministerio del Interior y Seguridad Pública Chile. Noveno Observatorio Chileno de Drogas, SENDA, 2011, Santiago, Chile.

Stewart, K., Silcock, D., \& Wegman, F. (2012). Reducing drink driving in low- and middle-income countries: Challenges and opportunities. Traffic Injury Prevention, 13, 93-95.

World Health Organization (WHO). (2009). Towards a global strategy to reduce harmful use of alcohol. Discussion paper for regional technical consultations on harmful use of alcohol. Geneva, Switzerland: Department of Mental Health and Substance Abuse, World Health Organization.

World Health Organization (WHO). (2010). Global strategy to reduce harmful use of alcohol. Geneva, Switzerland: World Health Organization.

World Health Organization (WHO). (2011). Global status report on alcohol and health 2011. Geneva, Switzerland: World Health Organization. 